\title{
The modernized treatment of hepatocellular cancer: time to think twice!
}

\author{
Jan Lerut
}

Published online: 26 July 2011

(C) Springer-Verlag 2011

\section{Introduction}

The field of hepatic oncology is rapidly changing due to the availability of a large diagnostic and therapeutic armamentarium.

The popularization of several loco-regional treatments (LRT) and the recent introduction in clinical practice of drugs such as tyrokinase inhibitors have changed the therapeutic algorithm of hepatocellular cancer (HCC). The role of surgery has been reduced markedly [1, 2].

This editorial aims at reminding surgical as well as medical communities that cure of liver cancer can only be obtained when resecting the tumor appropriately and that (long-term) results can be further improved when adhering to the classical oncologic paradigms.

\section{HCC in cirrhotic liver}

Up to the 1980s, treatment of HCC was very heterogeneous and even hazardous, mainly explained by the underlying hepatopathy which is present in $90 \%$ of patients. From then onwards, the outlook for patients with HCC changed due to major improvements in conventional as well as in transplantation surgery [2]. Hasegawa, Ton That Tung, Makuuchi, Nimura, Bismuth, Starzl and others were the

J. Lerut

Starzl Unit Abdominal Transplantation, University Hospitals

St. Luc, Université catholique de Louvain, UCL,

Brussels, Belgium

J. Lerut $(\bowtie)$

Cliniques Universitaires Saint-Luc, 10 Avenue Hippocrate,

1200 Brussels, Belgium

e-mail: jan.lerut@uclouvain.be major architects of many ingenious surgical procedures. Continuous expansion of technical possibilities allowed to perform more frequently partial liver resection (LR) and liver transplantation (LT). In 1983, the immunosuppressive drug cyclosporine moved LT from the experimental to the clinical arena. As a consequence, desperate, unresectable, tumor cases were sent for "last chance" LT.

The first wave of enthusiasm for LR and LT ebbed rapidly, especially in the western countries, due to the very high incidence of tumor recurrence. This could be easily explained for LR by the fact that the underlying, carcinogenic, territory (the remaining liver) remained in place, triggering the development of new tumors (estimated at $65 \%$ after 3 years) and for LT by the absence of selection criteria. The question about the place of LR and especially of LT in oncology was thereby answered. Bad results and substantial morbidity and mortality of all surgical procedures, related to liver failure and immunosuppressive therapy, induced the development of loco-regional tumor treatments (LRT) in the 1990s [3]. LRT includes percutaneous ethanol injection (PEI), radiofrequency destruction (and not ablation!) (RF), transarterial chemo-embolization (TACE) or embolization (TAE), transarterial radio-embolization (TARE), and combinations of these measures.

This second wave of enthusiasm in the treatment of HCC was amplified by advances in the field of imaging and engineering of technical devices. The "easy" applicability of LRT hindered the access of many patients to life-saving surgical procedures, this despite the fact that LRT has never been shown to cure HCC. Unfortunately the medical community has been, and still is, misled by the fact that most studies about LRT are done in an uncontrolled way and lack not only long-term overall (OS) but most importantly also disease-free survival (DFS) rates. It is well documented that 3-year OS for $\mathrm{HCC}$ is very similar 
whatever surgical or LRT therapy has been applied. It cannot be stressed enough that the final aim of any oncologic treatment must be DFS, an endpoint that unfortunately has been out of scope when promoting LRT, and that the gold standard to which any treatment should be compared is the pathology of the total hepatectomy specimen. Analysis of whole livers from patients previously treated with LRT has confirmed the shortcomings of LRT. The tumor is very often destroyed partially or subtotally but almost never "ablated" - and the longer the delay between LRT and LT, the more viable tumor is present!

The recent introduction of antitumor drugs represents the third wave of enthusiasm in the treatment of HCC. Undoubtedly the use of tyrokinase and angiogenic inhibitors has to be seen as a step forward, but unfortunately the big hype around the launch of these drugs further obscured the treatment of HCC. Indeed, the EASL and the recently updated AASLD guidelines on the treatment of $\mathrm{HCC}$ in cirrhotic patients have reduced surgery almost to a thirdrank player-only a minority of patients, presenting with an "early tumor", are considered for potentially curative LR or LT!

The access to multimodal LRT, different drug regimens, and surgical procedures curiously leads again to heterogeneous, less optimal care-nihil nove sub sole! Real progress should be based on the adherence to basic oncologic principles linking best surgery to best neo-adjuvant and adjuvant LRT and medical therapy...just as is done in all other fields of (GI tract) oncology.

Partial and total liver resection (this means LT) should be seen as complementary, and not as concurrent, therapies offering long-term DFS rates. In contrast to what is proposed in the "Barcelona algorithm", surgery should also be offered to intermediate HCC. Several western and many Asian teams treat HCC way beyond EASL/AASLD criteria: large HCC (30-75\%), multinodular HCC (25-43\%), and HCC with major vascular (3-15\%) and even bile duct (2\%) invasion. 5-year DFS rates of 20-30\% can be obtained in all these tumor constellations [4, 5]. All these patients would have gone into the palliative study arms in the EASL/AASLD guidelines [2]. It has thus been shown that LR can be useful in these intermediate HCC on the condition that surgery is adapted to the patient's condition, to the liver function, and to the intra-operative findings. As an example, HCC with bile duct invasion is best treated with en bloc bile duct and liver resection.

The same reasoning holds true for LT. The introduction in 1995 of the Milan criteria (MC), i.e., three tumors $<3 \mathrm{~cm}$ or one tumor $<5 \mathrm{~cm}$, represented a breakthrough in transplant oncology. 5-year DFS after LT reached $85 \%$ when adhering to the MC. Several excellent, western as well as eastern, experiences showed that the MC were too restrictive, unjustifiably denying access to LT to many patients. The UCSF group was the first one to expand the MC. Afterwards the Tokyo group broadened the criteria to five tumors and the Kyoto group went up to ten tumors on condition that tumor biology (using DCP determination) was favorable. A most reasonable expansion of transplant criteria is proposed by the Seoul group, expanding LT access to patients harboring up to six tumors, the biggest being $<6.5 \mathrm{~cm}$. All these Asiatic centers have the great advantage of counteracting the "factor time" of HCC by the use of living-donor LT [5].

When expanding the inclusion criteria for LT, one should take care not to transform "drop-out" before LT (on the waiting list) into a high recurrence rate after LT. Mazzaferro and Majno [1] warned of this by launching the "Metroticket" concept: the further you go (the larger the inclusion criteria), the greater the price (the higher the number of recurrences)! The "up to 7" score, consisting of the sum of the largest tumor diameter and the number of tumors, represents a good guideline for the clinician in relation to the indication for $\mathrm{LT}$ in $\mathrm{HCC}$. If the patient is initially outside the LT criteria, repetitive and aggressive LRT should be undertaken to downstage the initial tumor burden, bringing the patient back to within LT criteria. The value of LR as a bridge to salvage or de principe LT remains debated. There is indication that laparoscopic LR is of great interest in potential liver recipients. Without any doubt, inclusion criteria will be further refined by introducing tumor marker dynamics, tumor volume, molecular, and genetic analyses. The more criteria are expanded, the more it is justified to apply the "sandwich concept": LT (and also LR) should be complemented by adjuvant and neo-adjuvant therapies. There are already indications that sorafenib and $\mathrm{m}$-Tor inhibitors are beneficial in the surgical treatment of advanced HCC and in the treatment of recurrence after surgery.

\section{HCC in normal liver}

Classically it is stated that HCC in a normal liver (NCHCC) should (only) be treated by LR. Modern liver surgery, taking into account residual functional liver volume and preparatory steps such as portal vein embolization, allows extensive R0 LR. The literature review shows that 5-year DFS in these, frequently young, patients reaches only $24-59 \%$ and rates of tumor recurrence, most frequently (albeit less than in cirrhotic patients) in the liver, ranges from 30 to $78 \%[6,7]$ ! Revisiting of LR in these patients is therefore warranted. Pathologic analysis identified the following risk factors of recurrence after LR:R1 resection and, in $\mathrm{R} 0$ resection specimens, the presence of intra-hepatic metastases (also referred to as daughters, satellite nodules or multiple tumors), tumor capsule and 
lymph node involvement, micro- and macro-vascular invasion, absence of tumor capsule, and peri-operative blood loss and blood product use. The knowledge of these risk factors should be of great help to identify NC-HCC patients who would benefit from LT [6].

The recent analysis from the ELT Registry has shown that excellent long-term DFS (60\% at 5 years) can be obtained in "non-resectable" NC-HCC (62 patients). The $\mathrm{MC}$, validated in cirrhotic patients, are not applicable to these NC-HCC as virtually all these patients have tumor diameter and numbers far exceeding MC limits. Tumor diameter and microvascular invasion were not identified as significant determinants of survival after LT, whereas lymph node and/or vascular invasion were the main predictors of recurrence and DFS after LT in NC-HCC patients (45\% 5-year survival vs. $59 \%$ in absence of invasion).

Similar observations were made in 43 patients who underwent "salvage" LT for intra-hepatic tumor recurrence after liver resection. The 5-year OS and DFS rates in this group were 58 and 48\%, respectively. Macrovascular invasion and a period of $<12$ months between previous partial resection and tumor recurrence were the significant risk factors for poor outcome. This short time span probably reflects a more aggressive tumor biology.

These data (submitted for publication) show that LT certainly has a role to play in the treatment of NC-HCC. Careful lifelong follow-up, using regular imaging, is thus necessary, as a life-saving transplant, instead of palliative medical treatment, can be offered!

Pathological assessment of the resected tumor and of the non-tumorous liver tissue should become the guide to identify those patients at high-risk tumor recurrence after LR as well as after LT.

\section{Conclusion}

Surgeons and physicians alike should think twice before excluding cirrhotic and even non-cirrhotic HCC patients from potentially curative surgery! Better screening of highrisk patients allows earlier diagnosis of liver tumors. LRT and antitumor drugs should transform the care of HCC from a heterogeneous into a multimodal, integrated approach including surgeons, hepatologists, gastroenterologists, internists, interventional radiologists, radiotherapists, and oncologists. All available therapies should be considered in order to optimize the care of liver tumor patients. Surgery and especially transplantation, if possible, is the mainstay to which all other therapies must be compared. Without any doubt, surgery has to be given a broader place again in the field of hepatic oncology, especially when taking into account 5- and 10-year diseasefree survival rates!

\section{References}

1. Bhoori S, Sposito C, Germini A, Coppa J, Mazzaferro V (2010) The challenges of liver transplantation for hepatocellular carcinoma on cirrhosis. Transpl Int 23:712-722

2. Bruix J, Sherman M (2011) American Association for the Study of Liver Diseases. Management of hepatocellular carcinoma: an update. Hepatology 53:1020-1022

3. Lencioni R, Crocetti L, De Simone P, Filipponi F (2010) Locoregional interventional treatment of hepatocellular carcinoma: techniques, outcomes and future prospects. Transpl Int 23:698-703

4. Capussotti L, Ferrero A, Vigano L, Polastri R, Tabone M (2007) Liver resection for HCC with cirrhosis: surgical perspectives out of EASL/AASLD guidelines. Eur J Surg Oncol

5. Hwang S, Moon BD, Lee SG (2010) Liver transplantation and conventional surgery for advanced hepatocellular carcinoma. Transpl Int 23:723-727

6. Mergenthal H, Porte R (2010) Liver transplantation for unresectable hepatocellular carcinoma in patients without liver cirrhosis. Transplant Int 23:662-667

7. Trevisani F, Frigerio M, Santi V, Grignaschi A, Bernardi M (2010) Hepatocellular cancer in non-cirrhotic liver: a reappraisal. Digestive Liver Dis 42:341-347 